TRANSACTIONS OF THE

AMERICAN MATHEMATICAL SOCIETY

Volume 350, Number 11, November 1998, Pages 4609-4621

S 0002-9947(98)01996-5

\title{
LOWER BOUNDS FOR DIMENSIONS OF REPRESENTATION VARIETIES
}

\author{
ANDY R. MAGID
}

\begin{abstract}
The set of $n$-dimensional complex representations of a finitely generated group $\Gamma$ form a complex affine variety $R_{n}(\Gamma)$. Suppose that $\rho$ is such a representation and consider the associated representation $A d \circ \rho$ on $n \times n$ complex matrices obtained by following $\rho$ with conjugation of matrices. Then it is shown that the dimension of $R_{n}(\Gamma)$ at $\rho$ is at least the difference of the complex dimensions of $Z^{1}(\Gamma, A d \circ \rho)$ and $H^{2}(\Gamma, A d \circ \rho)$. It is further shown that in the latter cohomology $\Gamma$ may be replaced by various proalgebraic groups associated to $\Gamma$ and $\rho$.
\end{abstract}

\section{INTRODUCTION}

Let $\Gamma$ be a finitely generated group. The set of ismorphism classes of simple $n-$ dimensional complex representations of $\Gamma$ has the structure of an algebraic variety, as we will review below. In the simplest cases (for example, for linearly reductive $\Gamma)$ this variety is just a finite set of isolated points. Otherwise, there will be representations $\rho$ which have curves of non-isomorphic representations passing through them; such a $\rho$ is said to be deformable, and this happens exactly when the dimension of the variety of isomorphism classes of representations has positive dimension at the isomorphism class of $\rho$. This dimension is related to the dimension of the cohomology $H^{1}(\Gamma, A d \circ \rho)\left(A d \circ \rho\right.$ is the action of $\rho$ on $M_{n}(\mathbb{C})$ via conjugation): the variety of ismorphism classes of simple representations is the set of $\mathbb{C}$ points of a scheme whose Zariski tangent space at the class of $\rho$ can be identified with this cohomology. If the cohomology group happens to vanish, then the class of $\rho$ is isolated in the scheme and hence in the variety of $\mathbb{C}$ points. But the converse is not true: it may happen that $H^{1}(\Gamma, A d \circ \rho)$ is non-zero but the class of $\rho$ is isolated anyway (an example is referenced below). In any event, considering the scheme shows that the dimension of $H^{1}$ is an upper bound for the dimension of the variety.

In this paper, we will show that dimension of cohomology groups also provide a lower bound for the dimension at the class of $\rho$, the lower bound being the difference in the dimensions of $H^{1}(\Gamma, A d \circ \rho)$ and $H_{a}^{2}(\Gamma, A d \circ \rho)$ (the subscript $a$ on $H^{2}$ refers to alternate cohomology). Some observations are in order: first, although the arguments presented in this paper are self contained, this result fits a fairly standard pattern in deformation theory, and the proof here follows closely a similar result in the deformation theory of Galois representations given by Mazur [Maz,

Received by the editors May 25, 1995 and, in revised form, November 25, 1996.

1991 Mathematics Subject Classification. Primary 20C15.

Key words and phrases. Finitely generated roups, linear representations, varieties, cohomolgy. Partially supported by NSA grant MDA904-92-H-3038. 
Proposition 2, p. 399]. Second, at least in the case of standard $H^{2}$, this same lower bound is an easy corollary of work of Goldman and Millson that studies representation varieties from a differential-geometric and complex analytic point of view ([GM1], [GM2] see below; the author is grateful to the referee of an earlier version of this paper for this remark). One consequence of the algebraic approach followed here (which is in any case more elementary than via the Goldman-Millson results) is that the cohomology $H^{i}(\Gamma, A d \circ \rho)$ can be replaced by that of various proalgebraic groups associated with $\Gamma$, which we denoted $H_{a}^{2}$ above. For the case of $H^{1}$ these are known to yield the same cohomology group, but for $H^{2}$ they may be proper subgroups, and hence produce a better lower bound. In any event, these alternative cohomologies are used to compute the example that concludes the paper. It is also an open question about whether the Goldman-Millson results can be used to obtain the more restrictive lower bound which uses the alternative cohomology.

We now review the basic concepts and constructions in representation varieties (see $[\mathrm{LM}]$ for details).

Throughout, $\Gamma$ denotes a finitely generated group with presentation

$$
\Gamma=\left\langle g_{1}, \ldots, g_{d} \mid s_{a}, \quad a \in \mathcal{A}\right\rangle .
$$

An $n$-dimensional representation of $\Gamma$ is a homomorphism

$$
\rho: \Gamma \rightarrow G L_{n}(\mathbb{C})
$$

and we denote the set of all such $R_{n}(\Gamma)$ and call it the variety of representations of $\Gamma$ of degree $n$. It is indeed a variety: we can identify $R_{n}(\Gamma)$ with the $d$-tuples $\left(A_{i}\right)$ of elements of $G L_{n}(\mathbb{C})$ satisfying $s_{a}\left(A_{1}, \ldots, A_{n}\right)=I_{n}$ for all $a \in \mathcal{A}$. One can also view $R_{n}(\Gamma)$ as the $\mathbb{C}$ points of the scheme $\operatorname{Hom}\left(\Gamma, G L_{n}(\cdot)\right)$. We also denote this scheme by $\mathcal{R}_{n}(\Gamma)$.

Both variety and scheme of representations are affine; we denote their respective coordinate rings $A_{n}(\Gamma)\left(=\mathbb{C}\left[R_{n}(\Gamma)\right]\right)$ and $\mathcal{A}_{n}(\Gamma)\left(=\mathbb{C}\left[\mathcal{R}_{n}(\Gamma)\right]\right)$. The quotient of $\mathcal{A}_{n}(\Gamma)$ by its nilradical is isomorphic to $A_{n}(\Gamma)$, and this nilradical can be non-zero.

There is a representation

$$
\mathcal{P}: \Gamma \rightarrow G L_{n}\left(\mathcal{A}_{n}(\Gamma)\right)
$$

which is universal with respect to representations

$$
\rho: \Gamma \rightarrow G L_{n}(B)
$$

in commutative $\mathbb{C}$ algebras $B$ : for any such $\rho$ there is a unique $\mathbb{C}$ algebra homomorphism $f_{\rho}: \mathcal{A}_{n}(\Gamma) \rightarrow B$ such that $\rho=G L_{n}\left(f_{\rho}\right) \circ \mathcal{P}$.

There is a representation

$$
P: \Gamma \rightarrow G L_{n}\left(A_{n}(\Gamma)\right)
$$

which has a similar universal property with respect to representations in reduced commutative $\mathbb{C}$ algebras.

We can apply this universal property to a representation $\rho \in R_{n}(\Gamma)$. Then there is a unique homorphism $f_{\rho}: \mathcal{A}_{n}(\Gamma) \rightarrow \mathbb{C}$ such that $\rho=G L_{n}\left(f_{\rho}\right) \circ \mathcal{P}$; the kernel $M_{\rho}$ of $f_{\rho}$ is a maximal ideal and the corresponding point in the variety $R_{n}(\Gamma)$ is $\rho$ itself. When $\rho$ is understood from context, we denote $M_{\rho}$ just by $M$ alone.

Two representations $\rho$ and $\rho^{\prime}$ in $R_{n}(\Gamma)$ are equivalent if there is $A \in G L_{n}(\mathbb{C})$ with $A^{-1} \rho A=\rho^{\prime}$. This conjugation action defines an action of $P G L_{n}(\mathbb{C})$ on $R_{n}(\mathbb{C})$; we denote the orbit of $\rho$ under the action by $\mathcal{O}(\rho)$. Every orbit $\mathcal{O}(\rho)$ is of course a 
quasi-affine subvariety of $R_{n}(\Gamma)$. It is closed exactly when $\rho$ is semisimple and has trivial stabilizer exactly when $\rho$ is simple.

A representation $\rho \in R_{n}(\Gamma)$ is simple if and only if its image spans $M_{n}(\mathbb{C})$, and it is easy to see from this characterization that the subset $R_{n}(\Gamma)^{s}$ of simple representations forms an open subvariety of $R_{n}(\Gamma)$. Thus we obtain a geometric quotient variety

$$
R_{n}(\Gamma)^{s} \rightarrow R_{n}(\Gamma)^{s} / P G L_{n}(\mathbb{C})=S_{n}(\Gamma)
$$

where the quotient map is locally trivial for the étale toplogy. The points of $S_{n}(\Gamma)$ correspond to the equivalence classes of simple representations, the class of the simple representation $\rho$ being denoted $[\rho]$. The fibre over $[\rho]$ is the orbit $\mathcal{O}(\rho)$.

We also have the categorical quotient variety

$$
R_{n}(\Gamma) \rightarrow R_{n}(\Gamma) / / P G L_{n}(\mathbb{C})=S S_{n}(\Gamma)
$$

whose points parametrize the closed orbits. Since these are precisely the semisimple ones, the points of $S S_{n}(\Gamma)$ correspond to the equivalence classes of semisimple representations, the class of the semisimple representation $\rho$ being denoted $[\rho]$. Then $\mathcal{O}(\rho)$ is the unique closed orbit in the fibre over $[\rho]$.

Let $\rho \in R_{n}(\Gamma)$. We can consider the tangent spaces at $\rho$ to $\mathcal{O}(\rho), R_{n}(\Gamma)$, and $\mathcal{R}_{n}(\Gamma)$; if $\rho$ is also semisimple (respectively simple) we also can consider the tangent spaces at $[\rho]$ to $S S_{n}(\Gamma)$ and $S_{n}(\Gamma)$. We always have

$$
T_{\rho}(\mathcal{O}(\rho)) \subseteq T_{\rho}\left(R_{n}(\Gamma)\right) \subseteq T_{\rho}\left(\mathcal{R}_{n}(\Gamma)\right)
$$

(the inclusions can be strict); and if $\rho$ is simple that

$$
T_{\rho}\left(R_{n}(\Gamma)\right) / T_{\rho}(\mathcal{O}(\rho))=T_{[\rho]}\left(S_{n}(\Gamma)\right)=T_{[\rho]}\left(S S_{n}\right) .
$$

If $A d \circ \rho$ denotes the action of $\Gamma$ on $M_{n}(\mathbb{C})$ by $A \mapsto \rho(\gamma) A \rho(\gamma)^{-1}$, then the above tangent spaces are described in part as follows: the orbit tangent space is given by coboundaries

$$
T_{\rho}(\mathcal{O}(\rho))=B^{1}(\Gamma, A d \circ \rho)
$$

and the scheme tangent space is given by cocycles

$$
T_{\rho}\left(\mathcal{R}_{n}(\Gamma)\right)=Z^{1}(\Gamma, A d \circ \rho)
$$

from which it also follows that

$$
B^{1}(\Gamma, A d \circ \rho) \subseteq T_{\rho}\left(R_{n}(\Gamma)\right) \subseteq Z^{1}(\Gamma, A d \circ \rho)
$$

and hence for simple $\rho$ that

$$
T_{[\rho]}\left(S_{n}(\Gamma)\right) \subseteq H^{1}(\Gamma, A d \circ \rho) .
$$

Again, the inclusions can be strict.

As noted, a general reference for the above cited facts, and for other information about representation varieties, is $[\mathrm{LM}]$.

From the above considerations, we have that the dimension of $R_{n}(\Gamma)$ at $\rho$ satisfies the following inequalities:

$$
\operatorname{dim}_{\mathbb{C}} T_{\rho}((\mathcal{O}(\rho))) \leq \operatorname{dim}_{\rho}\left(R_{n}(\Gamma)\right) \leq \operatorname{dim}_{\mathbb{C}} T_{\rho}\left(\mathcal{R}_{n}(\Gamma)\right) .
$$

Note that since the coordinate ring of $R_{n}(\Gamma)$ is that of $\mathcal{R}_{n}(\Gamma)$ modulo nilpotents, which doesn't effect (Krull) dimension, we also have that $\operatorname{dim}_{\rho}\left(R_{n}(\Gamma)\right)=$ $\operatorname{dim}_{\rho}\left(\mathcal{R}_{n}(\Gamma)\right)$. 
We adopt the convention that $d^{*}(\cdot, \cdot)=\operatorname{dim}_{\mathbb{C}} D^{*}(\cdot, \cdot)$ for $D=B, Z, H$ so that the inequalities can be rewritten as

$$
b^{1}(\Gamma, A d \circ \rho) \leq \operatorname{dim}_{\rho}\left(R_{n}(\Gamma) \leq z^{1}(\Gamma, A d \circ \rho) .\right.
$$

The main result of this paper will be to show the following lower bound for the dimension:

$$
\operatorname{dim}_{\rho}\left(R_{n}(\Gamma)\right) \geq z^{1}(\Gamma, A d \circ \rho)-h^{2}(\Gamma, A d \circ \rho) .
$$

It then follows from the previous inequality that if $\rho$ is simple that

$$
h^{1}(\Gamma, A d \circ \rho) \geq \operatorname{dim}_{[\rho]}\left(S_{n}(\Gamma)\right) \geq h^{1}(\Gamma, A d \circ \rho)-h^{2}(\Gamma, A d \circ \rho) .
$$

We will also show how to make similar calculations using the cohomology of various proalgebraic hulls of $\Gamma$ and consider some applications.

We conclude this section by noting how the lower bound result, in the case of the standard cohomology, although it does not seem to have been previously remarked in the literature, could easily be obtained as a corollary of the work of Goldman and Millson. As previously mentioned, thanks are due to a referee for this argument and the references. In [GM2, Theorem 3.8, p. 349], it is shown that a certain deformation functor associated to a differential graded complex Lie algebra can be identified with the fibre of a certain analytic map from $H^{1}$ to $H^{2}$ (Lie algebra cohomology) called the Kuranishi map; then standard analysis implies that the fibre has dimension at least the difference of the dimensions of the cohomology groups. In [GM1, Theorem 6.8, p.82], it is shown that the analytic germ of the representation variety at a given representation prorepresents a deformation functor associated to a certain differential graded Lie algebra to which the above theorem then applies. (Both results are more general than the summary given here.) Thus it follows that at a given representation the representation variety is locally analytically isomorphic to the fibre of an analytic map between an $H^{1}$ and an $H^{2}$. Moreover, the cohomology can be seen to be equivalent to $H^{1}(\Gamma, A d \circ \rho)$ and $H^{2}(\Gamma, A d \circ \rho)$. Thus the lower bound formula in the standard cohomology case obtains. As mentioned above, whether these results also imply the formula for the alternative $H^{2}$ is not known, and even in the standard case the present paper is a more elementary algebraic path to the lower bound.

\section{LOCAL REPRESENTATIONS}

In this section, we consider representations of $\Gamma$ over (complete) local $\mathbb{C}$ algebras with residue field $\mathbb{C}$. We will make the convention that if $B$ is such a local ring then $M_{B}$ (or just $M$, if $B$ is understood) denotes its maximal ideal. If we have such a $B$ and a representation $\Gamma \rightarrow G L_{n}(B)$ then the residual representation $\Gamma \rightarrow G L_{n}(\mathbb{C})$ is the composite $\Gamma \rightarrow G L_{n}(B) \rightarrow G L_{n}(B / M=\mathbb{C})$.

We recall that our goal is to calculate $\operatorname{dim}_{\rho}\left(R_{n}(\Gamma)\right)$, which coincides with the dimension $\operatorname{dim}_{\rho}\left(\mathcal{R}_{n}(\Gamma)\right)$ and that the latter is the height of the maximal ideal $M=$ $M_{\rho}$ in the ring $\mathcal{A}=\mathcal{A}_{n}(\Gamma)$, which is the same as the Krull dimension of the local ring $\mathcal{A}_{M}$. In turn, this is the Krull dimension of the completion $\hat{\mathcal{A}}_{M}=\operatorname{proj} \lim \mathcal{A} / M^{i}$. Thus it will suffice to consider this latter ring. For convenience, we introduce the following terminology for it:

Definition 1. Let $\rho: \Gamma \rightarrow G L_{n}(\mathbb{C})$ be a representation. Then $\hat{A}(\rho)$ denotes the completion of $\mathcal{A}_{n}(\Gamma)$ at $M_{\rho}$. When $\rho$ is understood, this completion is denoted $\hat{A}$. We denote the maximal ideal of $\hat{A}(\rho)$ by $\hat{M}(\rho)$, or just by $\hat{M}$ when $\rho$ is understood. 
The homorphism $\mathcal{A}=\mathcal{A}_{n}(\Gamma) \rightarrow \hat{A}$ and the universal representation $\mathcal{P}$ induce a representation $\Gamma \rightarrow G L_{n}(\mathcal{A}) \rightarrow G L_{n}(\hat{A})$ which we denote $\tilde{\rho}$.

As noted, our goal is to bound the Krull dimension of $\hat{A}$. We first note that we have already determined an upper bound:

Proposition 2. Fix $\rho \in R_{n}(\Gamma)$. Then

$$
\operatorname{dim}\left(\hat{M} / \hat{M}^{2}\right)=z^{1}(\Gamma, A d \circ \rho) \quad \text { and } \quad \operatorname{dim}(\hat{A}) \leq z^{1}(\Gamma, A d \circ \rho) .
$$

Proof. As with every local ring, we have $\operatorname{dim}(\hat{A}) \leq \operatorname{dim}\left(\hat{M} / \hat{M}^{2}\right)$, where the latter is vector space dimension over the residue field $\mathbb{C}$. Since $\hat{A}$ is the completion of the finitely generated $\mathbb{C}$ algebra $\mathcal{A}_{n}(\Gamma)$ at $M=M_{\rho}$, we have that $M \subset \hat{M}$ and an induced isomorphism $M / M^{2} \rightarrow \hat{M} / \hat{M}^{2}$. Now $M / M^{2}$ is the $\mathbb{C}$ dual of the tangent space $T_{\rho}\left(\mathcal{R}_{n}(\Gamma)\right)$, and as previously noted this space is isomorphic to $Z^{1}(\Gamma, A d \circ \rho)$ whose dimension is $z^{1}(\Gamma, A d \circ \rho)$.

We then begin our investigation of a lower bound for this Krull dimension with a direct representation theoretic characterization of $\hat{A}$ :

Proposition 3. (Universal property of $\hat{A}(\rho)$ ) Fix $\rho \in R_{n}(\Gamma)$. Suppose $B$ is a complete local $\mathbb{C}$ algebra with $B / M_{B}=\mathbb{C}$ and that $\rho_{1}: \Gamma \rightarrow G L_{n}(B)$ is a representation with residual representation $\rho$. Then there exists a unique local $\mathbb{C}$ algebra homomorphism $\hat{f}: \hat{A} \rightarrow B$ such that $\rho_{1}=G L_{n}(\hat{f}) \circ \tilde{\rho}$.

Proof. The proof is basically to show that the universal property of $\mathcal{A}_{n}(\Gamma)$ passes to its completion: We know that there are unique homomorphisms $f_{1}: \mathcal{A}=\mathcal{A}_{n}(\Gamma) \rightarrow$ $B$ and $f: \mathcal{A} \rightarrow \mathbb{C}$ such that $\rho_{1}=G L_{n}\left(f_{1}\right) \mathcal{P}$ and $\rho=G L_{n}(f) \mathcal{P}$. Let $p: B \rightarrow B / M_{B}$ be the canonical projection. Then by assumption we have $\rho=G L_{n}(p) \rho_{1}$ so $\rho=$ $G L_{n}\left(p f_{1}\right) \mathcal{P}$ and hence $f=p f_{1}$. So the kernel of $p f_{1}$ contains, and thus coincides with, the kernel of $f$, which is the maximal ideal $M=M_{\rho}$. Thus $f_{1}(M) \subset M_{B}$. Then we have induced homomorphisms $\mathcal{A} / M^{i} \rightarrow B / M_{B}^{i}$ for each $i$ which induces a homomorphism $\hat{f}: \hat{\mathcal{A}} \rightarrow B$ on the completions. If $j: \mathcal{A} \rightarrow \hat{A}$ is the canonical injection, then $\hat{f} \circ j=f_{1}$, from which it follows both that $\rho_{1}=G L_{n}(\hat{f}) \circ \tilde{\rho}$ and that $\hat{f}$ is uniquely determined by this equation.

Proposition 3 implies a universal role for $\tilde{\rho}(\Gamma)<G L_{n}(\hat{A})$ which we now record for latter reference. We recall that if $B$ is a complete local $\mathbb{C}$ algebra of essentially finite type with residue field $\mathbb{C}($ such as $\hat{A})$ then $G L_{n}(B)=\operatorname{proj} \lim G L_{n}\left(B / M^{i}\right)$ is an inverse limit of algebraic groups and hence a proalgebraic group. We can also consider the Zariski closure of $\tilde{\rho}(\Gamma)$ in $G L_{n}(\hat{A})$. Then we have:

Remark 3.1. Let $\rho: \Gamma \rightarrow G L_{n}(\mathbb{C})$ and let $G_{\rho}(\Gamma)$ denote the Zariski closure of $\tilde{\rho}(\Gamma)$ in $G L_{n}(\hat{A}(\rho))$. Suppose $B$ is a complete local $\mathbb{C}$ algebra of essentially finite type with residue field $\mathbb{C}$ and $\rho_{1}: \Gamma \rightarrow G L_{n}(B)$ a representation with residual representation $\rho$. Then there is a unique (pro)algebraic group morphism $G_{\rho}(\Gamma) \rightarrow$ $G L_{n}(B)$ extending $\rho_{1}$, namely the restriction of $G L_{n}(\hat{f})$ of Proposition 3 to $G_{\rho}$.

A special case of complete local $\mathbb{C}$ algebras with residue field $\mathbb{C}$ are those of finite length, namely those with nilpotent maximal ideal. Since the complete local algebras we are considering are inverse limits of these, it will be necessary to understand representations into these finite length algebras and their lifts. Actually, it will be sufficient to look at the special case of surjections with kernel annihilating 
the maximal ideal, as we do in the next three results. We begin by stating some simple facts about the corresponding map on general linear groups.

Lemma 4. Let $p: B \rightarrow A$ be a local surjection of local $\mathbb{C}$ algebras with kernel $J$ and residue field $\mathbb{C}$. Then $G L_{n}(p): G L_{n}(B) \rightarrow G L_{n}(A)$ is surjective with kernel $I+M_{n}(J)$ (where $M_{n}(J)$ denotes the $n \times n$ matrices over $J$ ). If further $J^{2}=0$, then $M_{n}(J) \rightarrow I+M_{n}(J)$ by $x \mapsto I+x$ is an isomorphism of $G L_{n}(A)$ modules. If further $J \cdot M_{B}=0$, then it is an isomorphism of $G L_{n}\left(\mathbb{C}=A / M_{A}\right)$ modules.

Proof. Since $p$ is local, $p^{-1}\left(M_{A}\right)=M_{B}$, which means that if $p(b)$ is a unit of $A, b$ is a unit of $B$. This implies the surjectivity of $G L_{n}(p)$. Its kernel is clearly $I+M_{n}(J)$ and if $J^{2}=0$, then $M_{n}(J)^{2}=0$ so that $x \mapsto I+x$ is an isomorphism. $G L_{n}(B)$ acts on $M_{n}(B)$ and on itself by conjugation preserving the ideal $M_{n}(J)$ and the normal subgroup $I+M_{n}(J)$, and $I+M_{n}(J)$ itself acts trivially, so the action factors through $G L_{n}(A)$, and in this action the isomorphism is of $G L_{n}(A)$ modules. If $J M_{B}=0$, then the conjugation action further factors through $G L_{n}\left(A / M_{A}\right)$ and hence the isomorphism is of $G L_{n}\left(A / M_{A}\right)$ modules.

Because of the isomorphism $M_{n}(J) \rightarrow I+M_{n}(J)$ we refer to the latter as a module.

We next want to further assume that we have a representation of $\Gamma$ involved also. As we will see, we also need to use trivial representations: if $V$ is a vector space over $\mathbb{C}$, we let ${ }_{t} V$ denote $V$ with the trivial $\Gamma$ action.

Lemma 5. Let $p: B \rightarrow A$ be a local homomorphism of local $\mathbb{C}$ algebras with residue field $\mathbb{C}$, let $J=\operatorname{Ker}(p)$ and assume $J \cdot M_{B}=0$. Let $\rho: \Gamma \rightarrow G L_{n}(A)$ be a representation and let $\bar{\rho}: \Gamma \rightarrow G L_{n}(A) \rightarrow G L_{n}(\mathbb{C})$ be the associated residual representation. Then $I+M_{n}(J)$ is a $\Gamma$ module via $\rho$ and $\bar{\rho}$ and there is an isomorphism

$$
I+M_{n}(J) \rightarrow(A d \circ \bar{\rho}) \otimes_{\mathbb{C}}{ }_{t} J
$$

Proof. By Lemma $4, M_{n}(J)=I+M_{n}(J)$ is a $G L_{n}(A)$ module and a $G L_{n}(\mathbb{C})$ module through $G L_{n}(A) \rightarrow G L_{n}\left(A / M_{A}\right)$, so it is a $\rho$ and $\bar{\rho}$ module by composition. The action of $G L_{n}(\mathbb{C})$ on $M_{n}(J)=M_{n}(\mathbb{C}) \otimes J$ is by conjugation on the first factor, so that the action of $\Gamma$ is via $A d \circ \bar{\rho}$ on the first factor, which gives the desired isomorphism.

Now we consider the question of lifting representations. Suppose we have a local homomorphism $p: B \rightarrow A$ and a representation $\rho: \Gamma \rightarrow G L_{n}(A)$ as in Lemma 5 . Under what conditions can we lift to a representation $\rho_{1}: \Gamma \rightarrow G L_{n}(B)$ such that $\rho=G L_{n}(p) \rho_{1}$ ?

This is actually a pretty standard construction in cohomology. We will consider this in a little detail since the nature of the construction will be relevant later. We begin with the extension (exact sequence) associated to $p$ :

$$
1 \rightarrow I+M_{n}(J) \rightarrow G L_{n}(B) \rightarrow G L_{n}(A) \rightarrow 1
$$

and then pull this back along $\rho: \Gamma \rightarrow G L_{n}(A)$ to get the extension (where the middle group is the fibre product)

$(E(p, \rho))$

$$
1 \rightarrow I+M_{n}(J) \rightarrow G L_{n}(B) \times_{G L_{n}(A)} \Gamma \rightarrow \Gamma \rightarrow 1
$$

Since there exists a lift $\rho_{1}: \Gamma \rightarrow G L_{n}(B)$ of $\rho$ exactly when the second factor projection

$$
G L_{n}(B) \times_{G L_{n}(A)} \Gamma \rightarrow \Gamma
$$


has a section, any such section is of the form $\gamma \mapsto\left(\rho_{1}(\gamma), \gamma\right)$ where $G L_{n}(p) \rho_{1}(\gamma)=$ $\rho(\gamma)$. Thus the existence of a lift is equivalent to the splitting of the exact sequence $E(p, \rho)$. When $I+M_{n}(J)$ is abelian, this is equivalent to the vanishing of the cohomology class $[E(p, \rho)]$ in $H^{2}\left(\Gamma, I+M_{n}(J)\right)$, and in the circumstances of Lemma 5 we have a nice description of the coefficients $I+M_{n}(J)$ also. We record the results of this discussion:

Proposition 6. Let $p: B \rightarrow A$ be a local homomorphism of local $\mathbb{C}$ algebras with residue field $\mathbb{C}$, let $J=\operatorname{Ker}(p)$ and assume $J \cdot M_{B}=0$. Let $\rho: \Gamma \rightarrow G L_{n}(A)$ be a representation and let $\bar{\rho}: \Gamma \rightarrow G L_{n}(A) \rightarrow G L_{n}(\mathbb{C})$ be the associated residual representation. Then there exists a representation $\rho_{1}: \Gamma \rightarrow G L_{n}(B)$ with $\rho=$ $G L_{n}(p) \rho_{1}$ if and only if the cohomology class $[E(p, \rho)]$ is trivial in $H^{2}(\Gamma, A d \circ \bar{\rho}) \otimes_{\mathbb{C}} J$.

Remark 6.1. Suppose now that we restrict the local homomorphisms $p: B \rightarrow A$ to lie in some subcategory (for example, finite dimensional $\mathbb{C}$ algebras, or even finite dimensional quotients of a fixed local algebra, such as $\hat{A}(\rho))$ so that the terms in the associated extension $E(p)$ all lie in some fixed subcategory (for example, algebraic groups over $\mathbb{C}$ ) and so that any representation $\rho: \Gamma \rightarrow G L_{n}(A)$ factors through some universal $\Gamma \rightarrow \mathcal{G}(\Gamma)$ (in the algebraic groups case $\mathcal{G}(\Gamma)$ could be the universal proaffine algebraic hull, or Hochschild-Mostow group, of $\Gamma[\mathrm{HM}])$. Then the existence of a lift of $\rho$ is equivalent to the existence of a lift of $\mathcal{G}(\rho): \mathcal{G}(\Gamma) \rightarrow G L_{n}(A)$, and the same reasoning as above shows that this is equivalent to the triviality of the extension

$(\mathcal{E}(p, \rho)) \quad 1 \rightarrow I+M_{n}(J) \rightarrow G L_{n}(B) \times_{G L_{n}(A)} \mathcal{G}(\Gamma) \rightarrow \mathcal{G}(\Gamma) \rightarrow 1$

which, in the presence of a suitable cohomology theory $\mathcal{H}$, is equivalent to the triviality of the class $[\mathcal{E}(p, \rho)]$ in $\mathcal{H}^{2}(\mathcal{G}(\Gamma), A d \circ \bar{\rho}) \otimes J$.

This remark is useful in cases where the cohomology $\mathcal{H}^{2}$ is smaller than the cohomology $H^{2}$, as we will see below.

\section{Commutative Algebra}

The purpose of this section is to record two simple facts from local ring theory which will be used below in the proof of the main theorem. In this section we fix the following notation: $F$ and $A$ are local $\mathbb{C}$ algebras with residue field $C$, and $p: F \rightarrow A$ is a surjective local homomorphism with kernel $I$.

Lemma A. Suppose that $F$ is a catenary domain of dimension $n$ and that

$$
\operatorname{dim}_{\mathbb{C}}\left(I / M_{F} I\right)=d
$$

Then $\operatorname{dim}(A) \geq n-d$.

Proof. Let $m=\operatorname{dim}(A)$ and let $P_{0} \subsetneq P_{1} \subsetneq \cdots \subsetneq P_{m}=M_{A}$ be a maximal chain of prime ideals of $A$. Let $Q_{i}=p^{-1}\left(P_{i}\right)$. Then $Q_{0} \subsetneq Q_{1} \subsetneq \cdots \subsetneq Q_{m}=M_{F}$ is a saturated prime chain in $F$. Since $P_{0}$ is minimal, $Q_{0}$ is minimal over $I$, so that the height of $Q_{0}$ is at most the miminal number of generators of $I$ by the Krull Theorem [Mat, Theorem 18, p. 77]. And the minimal number of generators of $I$ is the dimension of $I / M_{F} I$, namely $d$. Since $F$ is catenary of dimension $n$ we have $n=m+\operatorname{height}\left(Q_{0}\right) \leq m+d$ so $m \geq n-d$. 
Lemma B. Assume that $I \neq 0$ and assume that $M_{F} / M_{F}^{2} \rightarrow M_{A} / M_{A}^{2}$ is an isomorphism (equivalently, that $I \subseteq M_{F}^{2}$ ). Then for any ideal $J \subsetneq I$ the exact sequence of $\mathbb{C}$ algebras

$$
0 \rightarrow I / J \rightarrow F / J \rightarrow A \rightarrow 0
$$

does not split as $\mathbb{C}$ algebras.

Proof. We can assume that $J$ is maximal in $I$ so that $I / J$ is a simple $F$ module and hence $F$ isomorphic to $F / M_{F}=\mathbb{C}$; say $I / J=\mathbb{C} x$. We also have that $M_{F}(I / J)=0$. Let $\bar{F}=F / J$, let $\bar{p}: \bar{F} \rightarrow A$ be the map induced from $p$, and suppose that we have a $\mathbb{C}$ algebra map $s: A \rightarrow \bar{F}$ such that $\bar{p} s=1_{A}$. We still have that $M_{\bar{F}} / M_{\bar{F}}^{2} \rightarrow M_{A} / M_{A}^{2}$ is an isomorphism, since $\bar{I}=I+J \subseteq M_{F}^{2}+J=M_{\bar{F}}^{2}$. Since $s$ has a left inverse, it carries non-units to non-units so that $s\left(M_{A}\right) \subseteq M_{\bar{F}}$. On the other hand, if $b \in M_{\bar{F}}$ then $b-s \bar{p}(b) \in \operatorname{Ker}(\bar{p})=\bar{I}$ so $M_{\bar{F}}=s\left(M_{A}\right)+\bar{I}$. Since $M_{\bar{F}} \bar{I}=0$, this description of $M_{\bar{F}}$ shows that $M_{\bar{F}}^{2}=s\left(M_{A}\right)^{2}=s\left(M_{A}^{2}\right)$. On the other hand, $s\left(M_{A}\right) \cap \bar{I}=0$ so $\bar{I} \cap s\left(M_{A}^{2}\right)=0$ so we would have $\bar{I} \cap M_{\bar{F}}^{2}=0$ which is a contradiction. Thus there is no such $s$.

\section{The Dimension Lower Bound}

We are now ready to establish the lower bound on the dimension of the representation variety. In view of Lemma A above, this will follow from the following theorem:

Theorem 7. Let $\rho \in R_{n}(\Gamma), \hat{A}=\hat{A}(\rho)$, and let $\hat{M}=M_{\hat{A}}$. Let $m=\operatorname{dim}_{\mathbb{C}}\left(\hat{M} / \hat{M}^{2}\right)$. Let $F=\mathbb{C}\left[\left[t_{1}, \ldots, t_{m}\right]\right]$ be a formal power series ring and let $F \rightarrow \hat{A}$ be a local surjection such that $M_{F} / M_{F}^{2} \rightarrow \hat{M} / \hat{M}^{2}$ is an isomorphism. Let I be its kernel. Then

$$
\operatorname{dim}_{\mathbb{C}}\left(I / M_{F} I\right) \leq h^{2}(\Gamma, A d \circ \rho) .
$$

Proof. We recall that we have a representation $\tilde{\rho}: \Gamma \rightarrow G L_{n}(\hat{A})$ whose residual representation is $\rho$. Let $J=I / M_{F} I$. Then $J$ is an $F / M_{F}=\mathbb{C}$ module. Choose $\phi: J \rightarrow \mathbb{C}, \mathbb{C}$ - (and hence $F-$ ) linear. We further assume that $\phi \neq 0$. Let $K \supseteq M_{F} I$ be the ideal such that $K / M_{F} I$ is the kernel of $\phi$. We consider the local surjections $p: F / M_{F} I \rightarrow \hat{A}$ and $\bar{p}: F / K \rightarrow \hat{A}$ whose kernels are $J$ and $\bar{J}=J / \operatorname{Ker}(\phi)$ respectively, and we consider the corresponding extensions $E(p)$ and $E(\bar{p})$. (Note that $\bar{J}=\mathbb{C}$.) From the commutative diagram

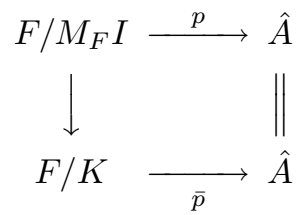

we have an induced map of the extension $E(p)$ to the extension $E(\bar{p})$ and we then have an induced map on cohomology $H^{2}\left(\Gamma, I+M_{n}(J)\right) \rightarrow H^{2}\left(\Gamma, I+M_{n}(\bar{J})\right)$ which carries $[E(p, \tilde{\rho})]$ to $[E(\bar{p}, \tilde{\rho})]$. Using Lemma 5 , this map on cohomology translates to the map

$$
1 \otimes \phi: H^{2}(\Gamma, A d \circ \bar{\rho}) \otimes J \rightarrow H^{2}(\Gamma, A d \circ \bar{\rho})
$$

so $[E(\bar{p}, \tilde{\rho})]=(1 \otimes \phi)[E(p, \tilde{\rho})]$. Recall that we are assuming that $\phi \neq 0$, which entails that $J \neq 0$ so that $I \neq 0$. We claim that this implies that both $[E(p, \tilde{\rho})] \neq 0$ 
and $[E(\bar{p}, \tilde{\rho})] \neq 0$, from which it will follow that the map $\phi \mapsto(1 \otimes \phi)([E(p, \tilde{\rho})])$ is injective.

For the claims, if $[E(p, \tilde{\rho})]=0$ then by Proposition 6 there is a representation $\rho_{1}: \Gamma \rightarrow G L_{n}\left(F / M_{F} I\right)$ lifting $\tilde{\rho}: \tilde{\rho}=G L_{n}(p) \rho_{1}$. By Proposition 3 applied to $\rho_{1}$, there is a unique local homomorphism $\hat{f}: \hat{A} \rightarrow F / M_{F} I$ such that $\rho_{1}=G L_{n}(\hat{f}) \tilde{\rho}$. It then follows from Proposition 3 applied to $\tilde{\rho}$ that $p \hat{f}=i d$, so that $p$ has a section. By Lemma $\mathrm{B}$, this means that $J=0$, which is a contradiction. The same argument implies the second claim: the universal property of $\hat{A}$ implies that $\bar{p}$ has a section, again contrary to Lemma B.

Thus we have an injective map $\operatorname{Hom}_{\mathbb{C}}(J, \mathbb{C}) \rightarrow H^{2}(\Gamma, A d \circ \rho)$, from which it follows that $\operatorname{dim}_{\mathbb{C}}(J) \leq h^{2}(\Gamma, A d \circ \rho)$.

Using Theorem 7 and Lemma A, we can produce the lower bound for the representation variety dimension:

Corollary 8. Let $\rho \in R_{n}(\Gamma)$ Then

$$
\operatorname{dim}_{\rho}\left(R_{n}(\Gamma)\right) \geq z^{1}(\Gamma, A d \circ \rho)-h^{2}(\Gamma, A d \circ \rho) .
$$

If $\rho$ is simple, then

$$
\operatorname{dim}_{[\rho]}\left(S_{n}(\Gamma)\right) \geq h^{1}(\Gamma, A d \circ \rho)-h^{2}(\Gamma, A d \circ \rho) .
$$

In either case, if $H^{2}(\Gamma, A d \circ \rho)=0$ then the inequality is an equality, $\rho$ (respectively $[\rho]$ ) is nonsingular on $R_{n}(\Gamma)$ (respectively $S_{n}(\Gamma)$ ), and the tangent space coincides with $Z^{1}(\Gamma, A d \circ \rho)$ (respectively $\left.H^{1}(\Gamma, A d \circ \rho)\right)$.

Proof. We use the notation of the statement of Theorem 7. By Lemma A, $\operatorname{dim}(\hat{A}) \geq$ $\operatorname{dim}(F)-\operatorname{dim}_{\mathbb{C}}\left(I / M_{F} I\right)$. By construction, $\operatorname{dim}(F)=\operatorname{dim}\left(\hat{M} / \hat{M}^{2}\right)$ and the latter is $z^{1}(\Gamma, A d \circ \rho)$ by Proposition 2. By Theorem $7, \operatorname{dim}\left(I / M_{F} I\right) \leq h^{2}(\Gamma, A d \circ \rho)$, and hence $\operatorname{dim}(\hat{A}) \geq z^{1}-h^{2}$. Since $\operatorname{dim}(\hat{A})=\operatorname{dim}_{\rho}\left(\mathcal{R}_{n}(\Gamma)\right)=\operatorname{dim}_{\rho}\left(R_{n}(\Gamma)\right)$, the first assertion obtains. Since $R_{n}(\Gamma)^{s} \rightarrow S_{n}(\Gamma)$ is locally trivial in the étale topology, if $\rho$ is simple we have $\operatorname{dim}_{[\rho]}\left(S_{n}(\Gamma)\right)=\operatorname{dim}_{\rho}\left(R_{n}(\Gamma)-\operatorname{dim}_{\rho}(\mathcal{O}(\rho))\right.$, where the latter is $b^{1}(\Gamma, A d \circ \rho)$. Thus $\operatorname{dim}_{[\rho]}\left(S_{n}(\Gamma)\right) \geq z^{1}-h^{2}-b^{1}=h^{1}-h^{2}$, giving the second assertion. And if $h^{2}(\Gamma, A d \circ \rho)=0$, then $\operatorname{since} \operatorname{dim}\left(T_{\rho}\left(\mathcal{R}_{n}(\Gamma)\right)=z^{1}(\Gamma, A d \circ \rho)\right.$ the lower bound implies that $\operatorname{dim}_{\rho}\left(R_{n}\right)=\operatorname{dim}_{\rho}\left(T_{\rho}\right)$ and hence that $R_{n}$ is nonsingular of dimension $z^{1}$ at $\rho$, and (using the local triviality in the étale topology) that $S_{n}$ is nonsingular of dimension $h^{1}$ at $[\rho]$.

Through any point $\rho$ in $R_{n}(\Gamma)$ we have the orbit $\mathcal{O}(\rho)$, which is a subvariety of dimension $b^{1}(\Gamma, A d \circ \rho)$. If we regard the representations in the orbit as trivially obtained from $\rho$, then truly new representations near $\rho$ are measured by $\operatorname{dim}_{\rho}\left(R_{n}(\Gamma)\right)-\operatorname{dim}_{\rho}(\mathcal{O}(\rho))$. Using Corollary 8, we then have:

Corollary 9. Let $\rho \in R_{n}(\Gamma)$. Then

$$
\operatorname{dim}_{\rho}\left(R_{n}(\Gamma)\right)-\operatorname{dim}_{\rho}(\mathcal{O}(\rho)) \geq h^{1}(\Gamma, A d \circ \rho)-h^{2}(\Gamma, A d \circ \rho) .
$$

Proof. We have by Corollary 8 and the above remarks that the left hand side of the inequality exceeds $z^{1}-h^{2}-b^{1}$, which equals the right hand side.

Corollary 9 shows that the main use of the inequality occurs in the cases were $h^{1}-h^{2} \geq 0$. We will see examples of this below.

The lower bound of Corollary 8 comes directly from the upper bound of Theorem 7. Thus a lower upper bound in the latter improves the lower bound in the former. 
We will obtain such using the technique discussed in Remark 6.1 ; namely, by noting that restricted cohomology theories apply here.

In the notation of Remark 6.1 , we note that since we are concerned only with the local surjection $F \rightarrow \hat{A}$ of Theorem 7, and the surjections $p: F / M_{F} I \rightarrow \hat{A}$ and $\bar{p}: F / K \rightarrow \hat{A}$ derived from it, the general linear groups involved are all proalgebraic (in fact the latter are all algebraic). Thus the representations of $\Gamma$ involved all factor through the Hochchild-Mostow group $G(\Gamma)$. Hence in the proof of Theorem 7 we can replace the ordinary group extensions $E(\cdot, \cdot)$ by the proalgebraic group extensions $\mathcal{E}(\cdot, \cdot)$ where the latter use the Hochschild-Mostow group $G(\Gamma)$ for $\mathcal{G}$ of 6.1. We note that these proalgebraic group extensions have (pro)unipotent kernels $\left(I+M_{n}(J)\right.$ in the notation of 6.1), and hence they admit sections as (pro)algebraic varieties. It follows that the extensions are classified by the corresponding cocycle classes in the proalgebraic group cohomology $H^{2}\left(G(\Gamma), I+M_{n}(J)\right)$ [DG, Proposition 2.3, p. 232]. Thus we obtain the analogue of Theorem 7 and its corollaries:

Corollary 10. In Corollary 8 and Corollary 9 we may replace $h^{2}(\Gamma, A d \circ \rho)$ by $h^{2}(G(\Gamma), A d \circ \rho)$.

This is actually an improvement: although $Z^{1}(\Gamma, \cdot)=Z^{1}(G(\Gamma), \cdot)$ and $B^{1}(\Gamma, \cdot)=$ $B^{1}(G(\Gamma), \cdot)$, so the two cohomologies coincide on the $H^{1}$ level, there is only an injection in general $H^{2}(G(\Gamma), \cdot) \rightarrow H^{2}(\Gamma, \cdot)$. And occasionally we also have some computational advantages in considering $H^{i}(G(\Gamma), \cdot)$.

We continue to analyze the proof of Theorem 7 . The key step (in the notation of that proof) is that the map $\phi \rightarrow(1 \otimes \phi)([E(p, \tilde{\rho})])$ is injective. It would be equally useful to know that the same is true of the (class of the) extension $\mathcal{E}(p, \sigma)$ where this is latter is derived from some factorization

$$
\Gamma \stackrel{j}{\longrightarrow} \mathcal{G}(\Gamma) \stackrel{\sigma}{\longrightarrow} G L_{n}(\hat{A})
$$

of $\tilde{\rho}$. But this is automatic: if the extension $(1 \otimes \phi)(\mathcal{E}(p, \sigma))=\mathcal{E}(\bar{p}, \sigma)$ is trivial, then $\sigma$ extends to $\sigma_{1}: \mathcal{G}(\Gamma) \rightarrow G L_{n}(F / K)$ and then $\rho_{1}=\sigma_{1} \circ j: \Gamma \rightarrow G L_{n}(F / K)$ lifts $\tilde{\rho}$, which leads to the same contradiction as in the original proof.

We take for $\mathcal{G}(\Gamma)$ the Zariski closure $G_{\rho}(\Gamma)$ of $\tilde{\rho}(\Gamma)$ in $G L_{n}(\hat{A})$ and for $\sigma$ the inclusion $G_{\rho}(\Gamma) \leq G L_{n}(\hat{A})$. Then we have the same result as Theorem 7 using instead the cohomology class $[\mathcal{E}(p, \sigma)] \in H^{2}\left(G_{\rho}(\Gamma), A d \circ \bar{\rho}\right) \otimes J$ (again proalgebraic group cohomology). Thus we conclude:

Corollary 11. In Corollary 8 and Corollary 9 we may replace $h^{2}(\Gamma, A d \circ \rho)$ by $h^{2}\left(G_{\rho}(\Gamma), A d \circ \rho\right)$.

This is again an improvement: as we will recall, the cohomology of a proalgebraic group is controlled by that of its prounipotent radical, and while the prounipotent radical of $G(\Gamma)$ can be enormous, that of $G_{\rho}(\Gamma)$ is always finitely generated.

Finally, we note that we could even take $\mathcal{G}(\Gamma)=G L_{n}(\hat{A})$ itself.

Corollary 12. In Corollary 8 and Corollary 9 we may replace $h^{2}(\Gamma, A d \circ \rho)$ by $h^{2}\left(G L_{n}(\hat{A}(\rho)), A d \circ \rho\right)$.

\section{Cohomology of Proalgebraic Hulls}

In this section, we make some calculations of $H^{*}(G, V)$ where $G$ is a proalgebraic group, especially $G=G(\Gamma)$ and $G=G_{\rho}(\Gamma)$ and $V$ is a $G$ module. 
We let $R_{u}(G)$ denote the prounipotent radical of $G$, and we assume that a maximal proreductive subgroup $P(G)$ of $G$ is selected, so that $G=R_{u}(G) \cdot P(G)$ (semi-direct product). Then there is a Hochschild-Serre spectral sequence

$$
H^{p}\left(G / R_{u}=P, H^{q}\left(R_{u}, V\right)\right) \Rightarrow H^{n}(G, V) ;
$$

since $H^{p}(P, \cdot)=0$ for $p>0$ the sequence collapses and we have

$$
H^{n}(G, V)=H^{n}(U, V)^{G} \text { where } U=R_{u}(G) .
$$

If further $V$ is semi-simple, so that $U$ acts trivally on $V$, then we have

$$
H^{n}(G, V)=\left(H^{n}(U, \mathbb{C}) \otimes_{\mathbb{C}} V\right)^{G} .
$$

The derived functor cohomology can be computed from the bar resolution; this identifies $H^{2}(U, V)$ with (algebraic) two cocycles [DG, Proposition 3.3, p. 235]. Such cocycles come from algebraic group extensions

$$
0 \rightarrow V \rightarrow E \rightarrow U \rightarrow 1
$$

which admit a morphic splitting $U \rightarrow E$ [DG, Proposition 2.3, p. 232]. But any extension (*) of proalgebraic groups has $E$ in fact prounipotent, since $V$ and $U$ are, and hence has a morphic splitting (since the corresponding extension

$$
0 \rightarrow V \rightarrow \operatorname{Lie}(E) \rightarrow \operatorname{Lie}(U) \rightarrow 0
$$

of linearly compact Lie algebras has a linear section). Thus $H^{2}(U, V)$ coincides with the classes of proalgebraic extensions of $U$ by $V$.

We recall from [LM, p. 72] that for any finite dimensional $\Gamma$ module $V$ there is a Grothendieck spectral sequence

$$
H^{p}\left(G(\Gamma), R^{q} F(V)\right) \Rightarrow H^{n}(\Gamma, V)
$$

where $F: \operatorname{Mod}(G(\Gamma)) \rightarrow \operatorname{Mod}(\Gamma)$ is the forgetful functor. The associated exact sequence of low degree terms is then

$$
\begin{gathered}
0 \rightarrow H^{1}(G(\Gamma), V) \rightarrow H^{1}(\Gamma, V) \rightarrow H^{0}\left(G(\Gamma), R^{1} F(V)\right) \\
\rightarrow H^{2}(G(\Gamma), V) \rightarrow H^{2}(\Gamma, V) .
\end{gathered}
$$

It is shown in [LM, p. 72] that $R^{1} F(V)=0$, from which it follows that $H^{1}(G(\Gamma), V)$ $\rightarrow H^{1}(\Gamma, V)$ is an isomorphism and that $H^{2}(G(\Gamma), V) \rightarrow H^{2}(\Gamma, V)$ is injective. These imply the following (in)equalities on dimensions:

$$
h^{1}(G(\Gamma), V)=h^{1}(\Gamma, V) \quad \text { and } \quad h^{2}(G(\Gamma), V) \leq h^{2}(\Gamma, V) .
$$

As noted above, this implies that Corollary 10 is an improvement on Corollaries 8 and 9 .

Now we want to turn to the case $G=G_{\rho}(\Gamma)$. Here we will write $U_{\rho}(\Gamma)$ in place of $R_{u}\left(G_{\rho}(\Gamma)\right.$ ) (and $U(\Gamma)$ for $R_{u}(G(\Gamma))$ ). Because $\Gamma \rightarrow G_{\rho}(\Gamma)$ is a group homomorphism from $\Gamma$ to a proalgebraic group, there is an induced proalgebraic group homomorphism $G(\Gamma) \rightarrow G_{\rho}(\Gamma)$, and since the above homomorphism has Zariski dense image the induced homomorphism is surjective. Thus we have a $\Gamma$ surjection $U(\Gamma) \rightarrow U_{\rho}(\Gamma)$ which induces a surjection on their abelianizations $U(\Gamma)^{\mathrm{ab}} \rightarrow U_{\rho}(\Gamma)^{\mathrm{ab}}$. A prounipotent group is finitely generated as such if its abelianization is [LM, Theorem 3.2, p. 88] which is the same as saying that its abelianization is finite dimensional. For cohomolgy calculations, we now compute the dimension of $U_{\rho}(\Gamma)^{\mathrm{ab}}$. 
Proposition 13. Let $\rho: \Gamma \rightarrow G L_{n}(\mathbb{C})$ be semi-simple. Then there is a surjection

$$
(A d \circ \rho)^{h} \rightarrow U_{\rho}(\Gamma)^{a b} \quad \text { where } h=h^{1}(\Gamma, A d \circ \rho) .
$$

In particular, $U_{\rho}(\Gamma)$ is finitely generated as a prounipotent group.

Proof. By definition, $G_{\rho}(\Gamma)$ is the Zariski closure of $\Gamma$ in $G L_{n}(\hat{A}(\rho))$, and it follows that $U=U_{\rho}(\Gamma)$ is a Zariski closed subgroup of the prounipotent group $K_{0}=$ $I+M_{n}(\hat{M})$, where $\hat{M}$ is the maximal ideal of $\hat{A}(\rho)$. Moreover, $K_{i}=I+M_{n}\left(\hat{M}^{i}\right)$ is a Zariski closed normal subgroup of $K_{0}$ with $K_{i-1} / K_{i}=A d \circ \rho \otimes \hat{M}^{i-1} / \hat{M}^{i}$ by Lemma 5 . It follows that all simple $\Gamma$ modules which occur in subquotients of $K_{0} / K_{i}$ are composition factors of $A d \circ \rho$. Since the $K_{i}$ are all normal in $K_{0}$ with $K_{0} / K_{i}$ affine it follows easily that $U=\bigcap U K_{i}$, with a similar formula holding for any closed subgroup of $U$. Now we consider the $\Gamma$ surjection $p: U(\Gamma)^{\mathrm{ab}} \rightarrow U^{\mathrm{ab}}$. The domain is the product of all simple $\Gamma$ modules, where the simple module $W$ occurs with multiplicity $h^{1}(\Gamma, W)$. Suppose that $W$ is not a composition factor of $A d \circ \rho$ and that $W$ has nonzero image under $p$. Then we have Zariski closed $\Gamma$ stable subgroups $L \supset M$ of $U$ with $M$ normal in $L$ and $L / M=W$. Since $L=\bigcap L K_{i}$ and $M=\bigcap M K_{i}$ (and $W$ is simple) there is an $i$ with $W \supset L K_{i} / M K_{i}$. But this then implies that $W$ is a composition factor of $K_{0} / K_{i}$, and hence of $A d \circ \rho$, contrary to hypothesis. It follows that the only simple modules in the product decomposition of $U^{\mathrm{ab}}$ which have nonzero image under $p$ are those occurring in $A d \circ \rho$, and of course each of these are direct factors of $A d \circ \rho$. Since the simple module $V$ occurs in the product with dimension $h^{1}(\Gamma, V)$ and $h^{1}(\Gamma, V) \leq h^{1}(\Gamma, A d \circ \rho)$ if $V$ is a direct factor of $A d \circ \rho$, the result follows.

\section{EXAMPLE}

This section considers an example, or rather a class of examples, which illustrates the use of the dimension lower bound. We fix the group $\Gamma$ throughout this section to be as follows: There is an exact sequence

$$
1 \rightarrow N \rightarrow \Gamma \rightarrow F \rightarrow 1
$$

where

1. $N$ is free abelian of rank 2 .

2. $F$ is finite.

3. $V=N \otimes_{\mathbb{Z}} \mathbb{C}$ is a simple $\Gamma$ module with $\Lambda^{2} V=\mathbb{C}$.

Let $W$ be a simple $F$ module regarded as a $\Gamma$ module with trivial $N$ action, and let $\rho$ be the corresponding representation.

In the notation of the preceding section, we then have that the unipotent radical $U(\Gamma)$ of $A(\Gamma)$ is isomorphic to $V$ as a $\Gamma$ module, so that (since $H^{1}(U(\Gamma), \mathbb{C})=$ $\left.\operatorname{Hom}(V, \mathbb{C})=V^{*}\right)$

$$
H^{1}(\Gamma, A d \circ \rho)=\operatorname{Hom}_{\Gamma}\left(V^{*}, \operatorname{End}(W)\right) .
$$

Also, since $H^{2}(U(\Gamma), \mathbb{C})=\operatorname{Hom}\left(\Lambda^{2} V, \mathbb{C}\right)$,

$$
H^{2}(A(\Gamma), A d \circ \rho)=\operatorname{Hom}_{\Gamma}\left(\Lambda^{2} V^{*}, \operatorname{End}(W)\right) .
$$

Now as a $\Gamma$ module $\operatorname{End}(W)=\mathbb{C} \oplus W_{1}$ where $W_{1}$ is a semi-simple module; since $V^{*}$ is simple we have that $\operatorname{Hom}_{\Gamma}\left(V^{*}, \operatorname{End}(W)\right)=\operatorname{Hom}_{\Gamma}\left(V^{*}, W_{1}\right)$ has dimension the multiplicity of $V^{*}$ in $W_{1}$. 
We assume further that $V^{*}$ is a composition factor of $W_{1}$ of multiplicity at least two. Since $W$ is simple, the multiplicity of $\Lambda^{2} V^{*}=\mathbb{C}$ in $\operatorname{End}(W)$ is one.

Then the basic inequality of Corollary 8 proves that $\rho$ has non-trivial deformations: $\left(S_{n}(\Gamma)\right.$ is positive dimensional at $\left.[\rho]\right)$.

\section{REFERENCES}

[DG] Demazure, M. and Gabriel, P., Introduction to Algebraic Geometry and Algebraic Groups, North-Holland, Amsterdam, 1980. MR 82e:14001

[GM1] Goldman, W. and Millson, J., The deformation theory of representations of fundamental groups of compact Kähler Manifolds, Pub. Math. IHES 67 (1988), 43-96. MR 90b:32041

[GM2] The homotpy invariance of the Kuranishi space, Ill. J. Math. 34 (1990), 337367. MR 91d:32025

[HM] Hochschild, G. and Mostow, G.D., Representations and representative functions of Lie groups, Ann. of Math. 66 (1957), 495-542. MR 20:5248

[LM] Lubotzky, A. and Magid, A., Varieties of Representations of Finitely Generated Groups, Mem. Amer. Math. Soc. 336, American Mathematical Society, Providence, 1985. MR 87c:20021

[LM2] Cohomology of unipotent and prounipotent groups, J. of Algebra 74 (1982), 76-95. MR 83d:14026

[Mat] Matsumura, H., Commutative Algebra, W. A. Benjamin Inc., New York, 1970. MR 42:1813

[Maz] Mazur, B., Deforming Galois representations, Galois Groups over $\mathbb{Q}$, MSRI Publ. 16, Springer-Verlag, New York, 1989, pp. 385-438. MR 90k:11057

Department of Mathematics, University of Oklahoma, Norman, Oklahoma 73019

E-mail address: amagid@ou.edu 